Chapter 7

\title{
Subclinical Hypothyroidism; Natural History, Long- Term Clinical Effects and Treatment
}

\author{
Jandee Lee and Woong Youn Chung \\ Additional information is available at the end of the chapter \\ http://dx.doi.org/10.5772/53688
}

\section{Introduction}

Subclinical hypothyroidism, defined as a mild degree of thyroid dysfunction biochemically, is a common clinical disease. It is marked by elevated serum concentrations of thyroid stimulating hormone (TSH) and normal serum concentrations of free thyroxine (fT4) and triiodothyronine (T3). Subclinical hypothyroidism can be divided into two categories, depending on the magnitude of the increase in serum TSH, with concentrations of $4.5-10 \mathrm{mU} / \mathrm{L}$ considered mild disease and $\mathrm{TSH}>10 \mathrm{mU} / \mathrm{L}$ considered severe disease. However, the definition and clinical significance of subclinical hypothyroidism are confounded by controversies over the exact upper limit of the reference range for se um TSH.

Subclinical hypothyroidism occurs in $4-20 \%$ of the adult population, a wide range that results from differences in age, gender, body-mass index, race, dietary iodine intake, and the cut-off concentrations of serum TSH used to define this condition. For example, most epidemiological surveys have reported that the prevalence of mild thyroid dysfunction was higher in older than in younger populations. In addition, overt or subclinical hypothyroidism occurs more frequently in areas of abundant iodine intake than in iodine-deficient areas, suggesting that iodine supplementation may increase the incidence of this disease. Subclinical hypothyroidism may be persistent or transient. In some of these patients, the transient expression of TSH-receptor blocking antibodies may indicate the recovery of thyroid function. Thus, it may be reasonable to reassess antibodies to thyroid hormones, including TSHreceptor blocking antibodies, in patients previously diagnosed with hypothyroidism to determine whether the latter is transient or permanent.

On average, $2-28 \%$ of patients with subclinical hypothyroidism progress to overt hypothyroidism, depending on age, gender, and the presence of anti-thyroid antibodies. However, baseline TSH concentration has been shown to be the most significant factor associated with 
progression to overt hypothyroidism. For example, a recent prospective study reported that the rate of overt hypothyroidism was about $10 \%$ in the entire study population, but was $2 \%$, $20 \%$, and $73 \%$ in patients with initial TSH concentrations of $5.0-9.9 \mathrm{mU} / \mathrm{L}, 10.0-14.9 \mathrm{mU} / \mathrm{L}$, and 15.0-19.9 $\mathrm{mU} / \mathrm{L}$, respectively.

Subclinical hypothyroidism may be associated with depressed systolic function at rest and left ventricular diastolic dysfunction at rest and during exercise. Persistent subclinical hypothyroidism may also affect the vascular smooth muscle cells, increasing systemic vascular resistance and arterial stiffness. Studies on the risks of cardiovascular disease and mortality due to coronary heart disease in these patients, however, have yielded conflicting results. To date, the clinical significance of cardiovascular effects after long-term subclinical hypothyroidism has not been definitively clarified.

The relationship between subclinical hypothyroidism and lipid metabolism is also unclear. Subclinical hypothyroidism has been associated with variable increases in total cholesterol and LDL-cholesterol, higher plasma concentrations of oxidized LDL-cholesterol, and inconsistent changes in serum concentrations of HDL-cholesterol. These lipid patterns, however, may also be affected by age, gender, smoking status, the cause and duration of thyroid dysfunction, and serum TSH concentration. All of these findings have suggested that there are no definitive TSH cutoffs for association with lipids.

Subclinical hypothyroidism may also be associated with mood disorders such as major depression. Moreover, the life-time prevalence of major depressive disorder may be greater in individuals with than without subclinical hypothyroidism. These findings, however, do not suggest a direct causal link between subclinical hypothyroidism and mood disturbance. Studies of the relationships between cognitive function and thyroid hormone levels within the normal reference range have produced somewhat conflicting results. Some studies have reported that higher TSH is associated with poorer cognitive function, whereas others found that higher TSH is correlated with better cognitive performance. These studies indicate that variations in serum TSH concentrations in individuals having normal thyroid hormone concentrations (fT4, T3) may be related to cognitive impairment, especially in older individuals. Further investigations of the relationships between serum TSH concentrations and mood disturbance or cognitive function may be required.

In this chapter, we summarize the definition, causes, diagnosis, and treatment of subclinical hypothyroidism. We also discuss the clinical effects of this illness, including the effects of long-term subclinical hypothyroidism on the cardiovascular system, lipid metabolism, mood disorders and cognitive function.

\section{Subclinical hypothyroidism}

\subsection{Definition}

The term subclinical indicates the presence of a disease without obvious symptoms, suggesting that the disease may be at an early stage. Subclinical thyroid disease is based on the deli- 
cate sensitivity of the hypothalamic-pituitary-thyroid (HPT) axis. Subclinical hyperthyroid and hypothyroid conditions are laboratory-based diagnoses. By definition, subclinical hypothyroidism is characterized by abnormal serum TSH and normal fT4 and T3 concentrations.

In 2002, a scientific review and consensus committee, which included representatives from the American Thyroid Association (ATA), the American Association of Clinical Endocrinologists (AACE), and the US Endocrine Society, convened a panel of experts to define subclinical thyroid disease, review the literature concerning the risks and benefits of treatment, and make recommendations about evaluation and population-based screening (Surks et al, 2004). This committee defined subclinical hypothyroidism as "a serum TSH concentration above the statistically defined upper limit of the reference range when serum free $\mathrm{T} 4$ concentration is within its reference range". However, the definition and the clinical significance of subclinical hypothyroidism are confounded by controversies over the correct upper limit of the reference range for serum TSH. Meticulous studies from the United States and elsewhere have addressed this reference range, taking into account the influence of the inclusion or exclusion of subjects with a personal or family history of thyroid disease and patients positive for antithyroid antibodies. Because subclinical hypothyroidism is only detected as an abnormal TSH concentration, defining the TSH reference range is critical. Circulating TSH is heterogeneous in glycosylation and biological activity. Assays vary widely because current TSH immunometric assays involve the use of monoclonal antibodies that differ in specificity and may therefore bind to different TSH isoforms. Therefore, variations in the reference intervals obtained with different methods reflect differences in epitope recognition of different TSH isoforms. Since these differences make it difficult to establish a universal upper TSH reference limit, the use of thyroid antibody tests has resulted in a progressive decrease in the upper limit of the normal range over the last decades, from 10.0-7.0 $\mathrm{mU} / \mathrm{L}$ to $4.0-5.0 \mathrm{mU} / \mathrm{L}$ (Biondi \& Cooper, 2008).

Lymphocytic infiltration of the thyroid gland is present in up to $40 \%$ of healthy women. Moreover, competitive immunoassays have shown that $10 \%$ of the general population is positive for antithyroglobulin antibody $(\mathrm{Tg} \mathrm{Ab})$ and $12 \%$ are positive for detectable antithyroid peroxidase (TPO) antibody levels (Biondi et al, 2005). Furthermore, ultrasound characteristics can reveal the typical pattern of hypoechogenicity, heterogeneity, and increased blood flow seen in autoimmune thyroiditis. A hypoechoic or irregular echo ultrasound pattern may precede positivity for TPO Abs in patients with autoimmune thyroid disease, and TPO Abs may not be detected in more than $20 \%$ of individuals with ultrasound evidence of thyroid autoimmunity (Vejbjerg et al, 2006). A high thyroid autoantibody titer (usually against TPO $\mathrm{Ab}$ and/or $\mathrm{Tg} \mathrm{Ab}$, or more rarely, TSH receptor $\mathrm{Ab}$ ) is frequently associated with a persistently elevated serum TSH concentration. Although the thyroid gland is usually goitrous, it may also be normal or atrophic, its hypoechogenicity at ultrasound evaluation could allow clinicians to identify individuals with subclinical hypothyroidism due to autoimmune disease (Biondi, 2012).

Therefore, it has been recommended that the reference serum TSH concentration be determined using blood sampled in the morning from fasting euthyroid subjects with no family history of thyroid disease, who are not taking any medication, have no visible or palpable 
goiter or pathological thyroid ultrasonography findings, and are not positive for TPO Ab or $\mathrm{Tg} \mathrm{Ab}$ (Kratzsch et al, 2005). Evidence from the National Health and Nutritional Examination Survey (NHANES III) of a large 'reference' population without evidence of thyroid disease indicated that $95 \%$ of adults have serum TSH concentrations of $0.45-4.12 \mathrm{mU} / \mathrm{L}$, indicating that the widely applied upper limit of normal for serum TSH of around $4.5 \mathrm{mU} / \mathrm{L}$ remains appropriate (Hollowell et al, 2001). A panel of experts has classified subjects with subclinical hypothyroidism into two categories, based on the degree of serum TSH elevation and pathophysiological consequences; these include patients with mild (4.5-10 mU/L) and more severely elevated (TSH>10 mU/L) serum TSH (Surks et al, 2004).

Subclinical hypothyroidism should be diagnosed only after a detailed personal and family history, pharmacological evaluation and an accurate clinical assessment. Some familial disorders, including a family history of autoimmune thyroid disease and/or endocrine or systemic autoimmune disorders, and genetic disorders such as Down, Turner and Klinefelter syndromes, should be investigated to identify subjects with an increased predisposition to developing autoimmune thyroiditis. In addition, a high thyroid autoantibody titer is frequently associated with a persistently elevated serum TSH concentration. The ultrasonographic hypoechogenicity of the thyroid gland can be used to identify individuals with subclinical hypothyroidism due to autoimmune disease (Wilson \& Curry, 2005).

Prior to treatment, patients should be evaluated for transient and false causes of mild increases in TSH. Reference TSH ranges by age, race and body mass index can help avoid misclassifying patients with increased serum TSH (Biondi, 2012). Interestingly, serum TSH concentrations are higher in white than in black populations, suggesting that TSH is influenced by genetics and ethnicity/race. Moreover, increased serum TSH might not always reflect mild thyroid hormone deficiency in elderly subjects from iodine sufficient areas because the distribution of serum TSH shifts to higher concentrations with age. An increased serum TSH concentration in older individuals may reflect recovery from acute illness or the use of drugs that can interfere with thyroid function (Surks et al, 2004). Since serum TSH concentrations are higher in overweight and obese individuals than in lean subjects, subclinical hypothyroidism may be falsely diagnosed in overweight and obese patients, especially in those negative for thyroid autoantibodies. However, this altered thyroid hormone pattern can be reversed by losing weight (Biondi, 2010).

\subsection{Causes}

The most frequent causes of subclinical hypothyroidism are autoimmune thyroiditis and previous treatment for hyperthyroidism. In about $60-80 \%$ of patients, subclinical hypothyroidism has been associated with TPO Abs, a marker of chronic lymphocytic (Hashimoto's) thyroiditis. Hashimoto's thyroiditis is more common in females than in males, with the overall incidence increasing with age in both sexes. Transient or persistent increases in serum TSH may occur after subacute, post-partum or painless thyroiditis and after an infiltrative disease (Riedel's thyroiditis, amyloidosis, hemochromatosis and cystinosis) or infectious disorder of the thyroid gland. Patients treated for overt thyroid failure often have subclinical hypothyroidism because of inadequate thyroid hormone supplementation, poor adherence, 
drug interactions, or inadequate monitoring of treatment (Cooper \& Biondi, 2012). Between $17.6 \%$ and $30 \%$ of patients with overt hypothyroidism were reported to have subclinical thyroid disease due to inadequate thyroid hormone supplementation (Canaris et al, 2000).

Subclinical hypothyroidism may also result from therapies that destroy thyroid tissue, such as thyroid surgery, radioiodine treatment or external radioiodine therapy. Mild, overt hypothyroidism frequently occurs after external radiotherapy of the head and neck area and develops gradually within the first year, with a risk that appears to be dose-dependent (Biondi \& Cooper, 2008). Radioiodine treatment of hyperthyroidism in patients with Graves' disease results in hypothyroidism in at least $50 \%$ of patients, depending on the dose administered. Partial thyroidectomy for hyperthyroidism or nodular goiter has been associated with a similar risk for the development of hypothyroidism. Subclinical hypothyroidism soon after radioiodine treatment or partial thyroidectomy may be a transient phenomenon, not always indicative of progressive or permanent hypothyroidism. Even in the absence of ablative thyroid treatment, it has been estimated that $5-20 \%$ of patients with Graves' disease will eventually develop hypothyroidism (Boelaert et al, 2009).

Other causes of subclinical hypothyroidism include autoimmune diseases, such as type I diabetes mellitus and Addison's disease. Down and Turner syndromes have also been associated with the development of overt and subclinical hypothyroidism. The risk of subclinical hypothyroidism during pregnancy is considerable in women identified during the first trimester as being positive for antithyroid antibodies (Wilson \& Curry, 2005). Non-thyroidal illness may be associated with a transient and modest increase in serum TSH, especially during the recovery phase, although in most of these patients an increase in TSH concentration reflects underlying thyroid disease. Several drugs, including iodine-containing compounds such as radiographic contrast agents, lithium carbonate, cytokines and interferon, may induce subclinical or overt hypothyroidism, particularly in patients with underlying autoimmune thyroiditis (Biondi, 2012).

It is necessary to distinguish subclinical hypothyroidism from other causes of physiological, artificial, or transiently increased serum TSH. Serum TSH concentrations should be measured 3-6 months later to rule out a laboratory error or a transient increase caused, for example, by drugs that interfere with thyroid function, by thyroiditis, and by possible toxic injury to the thyroid gland (Cooper \& Biondi, 2012). Serum TSH concentrations are higher in healthy elderly than in healthy young people because of a shift in TSH distribution with age. Rarely, laboratory patterns indistinguishable from subclinical hypothyroidism are observed in patients with TSH-receptor mutations causing mild TSH resistance, mutations that can affect up to $0.6 \%$ of Caucasian people. Clues to the presence of this disorder are a family history of increased serum TSH concentrations and an absence of thyroid autoimmunity (Jordan et al, 2003). Serum TSH concentrations are higher in overweight and obese individuals, which may result in a false diagnosis of subclinical hypothyroidism. Mild increases in serum TSH concentration in obese people are usually associated with serum T3 concentrations at the upper limit of the normal range. The latter may be due to increased de-iodinase activity, which acts as a compensatory mechanism during fat accumulation to increase energy expenditure. This altered thyroid hormone pattern can be reversed by weight loss. Table 1 shows 
the comparison of causes between subclinical hypothyroidism and elevated serum TSH concentrations that are not associated with persistent subclinical hypothyroidism (Biondi \& Cooper, 2008) (Cooper \& Biondi, 2012).

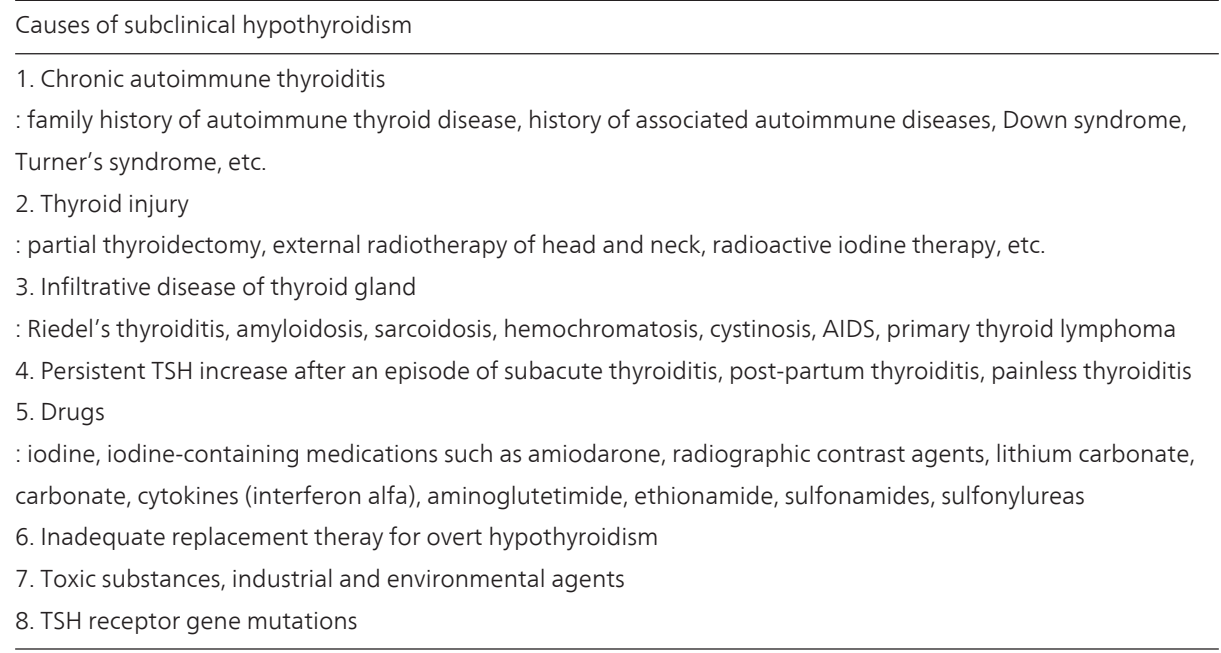

Causes of elevated serum TSH concentrations that are not associated with persistent subclinical hypothyroidism

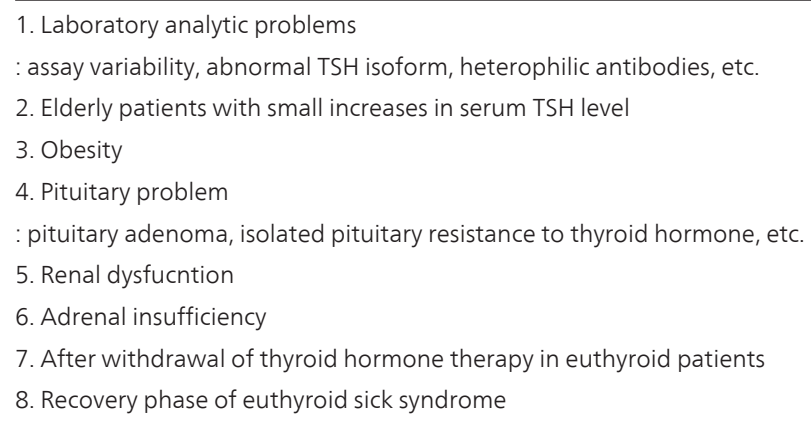

Table 1. Causes of elevated serum TSH concentration

\subsection{Prevalence and natural history}

The prevalence of subclinical hypothyroidism in adults has been reported to range from $4 \%$ to $20 \%$ (Cooper \& Biondi, 2012). This wide range reflects important differences in race and dietary iodine intake, as well as patient age, gender, body mass index and differences in TSH evaluation methods. Large, population-based screening studies, including the Whickham Survey (Tunbridge et al, 1977), NHANES III (Hollowell et al, 2001), and the Colorado thyroid prevalence study (Canaris et al, 2000), have provided impor- 
tant epidemiological data about subclinical hypothyroidism. According to the Whickham Survey, which defines subclinical hypothyroidism as serum TSH concentrations $>6$ $\mathrm{mU} / \mathrm{L}$, this condition was present in $7.5 \%$ of females and $2.8 \%$ of males. TSH concentrations did not vary with age in males but increased markedly in females after age 45 years. Serum TSH concentration was not age-related in women negative for antithyroid antibodies. The NHANES III study, which defined subclinical hypothyroidism as serum $\mathrm{TSH}>4.6 \mathrm{mU} / \mathrm{L}$, reported that $4.3 \%$ of individuals had subclinical hypothyroidism. TPO Abs were significantly associated with hypothyroidism, were more prevalent in women than in men, increased with age, and were more prevalent in whites than in blacks. The Colorado study, which defined subclinical hypothyroidism as serum TSH $>5.1 \mathrm{mU} / \mathrm{L}$, showed that serum TSH concentrations were elevated in $9.5 \%$ of subjects, and that the percentage increased with each decade of age in women but not in men. All of these surveys reported that the prevalence of subclinical hypothyroidism was higher in older populations. This disease is more common in iodine-sufficient than in iodine-deficient countries, suggesting that iodine supplementation may increase its incidence.

The natural history of subclinical hypothyroidism depends on the underlying cause and the characteristics of each patient. However, long-term outcome data on patients with subclinical hypothyroidism are rare, making it difficult to define the definitive course of this disease. Subclinical hypothyroidism may be progressive or reversible. With a progression rate of only $5 \%$ per year, it is reasonable to assume that, especially in patients with serum TSH concentrations $\leq 10 \mathrm{mU} / \mathrm{L}$, subclinical hypothyroidism may not be caused by the progression of any specific disease state (Wilson \& Curry, 2005). One large follow up study showed that, of patients with modestly elevated serum TSH concentrations, $60 \%$ show a spontaneous return to the reference range during a mean 5 year follow-up period (Meyerovitch et al, 2007). Moreover, many patients with non-autoimmune thyroiditis may develop transient TSH elevation.

In a significant number of patients, especially those who are older, female, and positive for TPO Abs, there is an increased risk of progression to overt hypothyroidism. The Whickham cohort survey, with a 20-year follow-up period, showed that the annual rate of progression from subclinical to overt hypothyroidism was $2.6 \%$ in patients negative for thyroid antibodies, but $4.3 \%$ if TPO Abs were present. Transient expression of TSH-receptor blocking antibodies may explain the improvements in thyroid function and the progressive TSH normalization that may be observed in some patients with autoimmune hypothyroidism. The annual rate of progression to overt disease was about $4 \%$ in women with increased serum TSH and positive for antithyroid antibodies, $2-4 \%$ in women with increased serum TSH concentrations alone, and 1-3\% in women with only antithyroid antibodies. Serum TSH concentrations tend to return to normal more frequently in people with concentrations of 4-6 $\mathrm{mU} / \mathrm{L}$, whereas TSH concentrations of $10-15 \mathrm{mU} / \mathrm{L}$ are associated with a reduced rate of normalization of thyroid function. The annual rate of progression to overt hypothyroidism in patients with subclinical hypothyroidism induced by radioiodine or surgery is $2-6 \%$. Pregnant women with Hashimoto thyroiditis also are at high risk of disease progression. High 
dose iodine intake is also associated with an increased risk of progression to overt hypothyroidism (Biondi \& Cooper, 2008).

The risk of progression from subclinical to overt hypothyroidism is less common, whereas the recovery of thyroid function is more common, in children and adolescents than in adults. Progression from mild to overt hypothyroidism may be related to the cause of thyroid hormone deficiency, the basal TSH concentration, and the age of the patient. Transient expression of TSH-receptor blocking antibodies may explain the recovery of thyroid function in some patients (Biondi \& Cooper, 2008). Patients previously diagnosed with subclinical hypothyroidism should be reevaluated to determine whether this condition is persistent. This may be accomplished by progressive reduction in LT4 dosage followed by serial TSH testing.

\subsection{Consequences of subclinical hypothyroidism}

Subclinical hypothyroidism may be associated with adverse cardiovascular events, cardiac dysfunction, lipid metabolism, and neuropsychiatric symptoms. To date, however, there is insufficient evidence regarding the association between long-term subclinical hypothyroidism and systemic sequelae.

\subsubsection{The cardiovascular system and the risk of heart failure}

Cardiovascular diseases are the most common causes of death worldwide, primarily affecting older adults. Abnormal TSH concentrations may be a novel cardiac risk factor. Even mildly altered thyroid status has been reported to affect serum cholesterol concentrations, heart rhythm and rate, ventricular function, risk of coronary artery disease, and cardiovascular mortality. Patients with subclinical hypothyroidism may have depressed systolic function at rest, and left ventricular diastolic dysfunction at rest and during exercise. Vascular function may also be impaired by thyroid hormone deficiency. However, the risks of cardiovascular and heart failure in patients with subclinical hypothyroidism remain unclear. Several large scale meta-analyses have assessed the risks of cardiovascular and all-cause mortality in patients with subclinical hypothyroidism. In one meta-analysis, cardiovascular mortality was higher in patients $<65$ years old with subclinical hypothyroidism, but not in older people (Razvi et al, 2008). A second meta-analysis confirmed that subclinical hypothyroidism was associated with modestly increased cardiovascular risks of coronary heart disease and total mortality (Ochs et al, 2008). In addition, reanalysis of the data from the Whickham Survey cohort showed that systolic and diastolic blood pressures and total cholesterol concentrations were higher in patients with subclinical hypothyroidism than in controls in a euthyroid state (Razvi et al, 2010). However, a large prospective cohort study of 559 subjects aged 85 year, including subclinical hypothyroidism was present in 30 patients and 21 individuals monitored for 4 years, provided opposite evidences. This study found that raised serum TSH concentrations was associated with decreased mortality in individuals older than 85 years, and this might be attributed to a lower metabolic rate. In this study, individuals with subclinial hypothyroidism had lower all-causes and cardiovascular mortality than clinically euthyroid individuals, although serum cholesterol levels were higher 
(Gussekloo et al, 2004). Similarly, other studies reported that subclinical hypothyroidism was not associated with increased overall mortality risk in elderly subjects. Crucially, a meta-analysis of individual participant data from 11 prospective cohort studies has shown no overall association of subclinical hypothyroidism with coronary heart disease events, mortality or total mortality (Rodondi et al, 2010). However, significant associations were observed when the degree of serum TSH elevation was stratified, in that coronary heart disease events and mortality risks were significantly increased in individuals with serum TSH $>10 \mathrm{mU} / \mathrm{L}$. This correlation with greater biochemical abnormality was consistent with studies in patients with heart failure, in that incident heart failure risk was evident, or of greater magnitude, when serum TSH was $>10 \mathrm{mU} / \mathrm{L}$. By contrast with studies suggesting that the risk of coronary heart disease decreased with patient age, findings of this meta-analysis showed no interaction between mortality due to coronary heart disease and age.

In summary, several prospective, population-based cohort studies found that subclinical hypothyroidism was associated with increased risks of cardiovascular disease and mortality, whereas other, similar studies showed no correlation between subclinical hypothyroidism and the incidence of and mortality due to coronary heart disease.

\subsubsection{Lipid profiles}

Thyroid hormones have varied effects on lipid metabolism, because thyroid function regulates cholesterol synthesis and degradation and mediates the activity of key enzymes in these pathways. Thyroid hormones reduce cholesterol concentration mainly through the increased expression of low-density lipoprotein (LDL)-cholesterol receptors in the liver and peripheral organs (Duntas \& Brenta, 2012). Overt hypothyroidism increases the serum concentrations of total and LDL-cholesterol, as well as altering the concentrations of other lipoproteins and apolipoproteins. In contrast, lipid changes in individuals with subclinical hypothyroidism are considerably less marked, with studies showing inconsistent results. For example, studies comparing lipid profiles in subclinical hypothyroid patients and euthyroid controls have found that subclinical hypothyroidism was associated with lipid abnormalities, especially increases in total and LDL cholesterol, but its effects on the concentrations of high-density lipoprotein (HDL)-cholesterol, triglycerides and lipoprotein(a) were unclear (Cappola \& Ladenson, 2003) (Duntas \& Wartofsky, 2007).

The United States Colorado study, with a population sample of 25,862 subjects, showed that patients with subclinical hypothyroidism had higher total cholesterol concentrations than euthyroid individuals (Canaris et al, 2000). In the NHANES III cohort, cholesterol and triglyceride concentrations were higher in patients with subclinical hypothyroidism than in euthyroid subjects, but these effects were no longer observed after adjusting for variables such as sex, race, age, and treatment with lipid lowering drugs (Hollowell et al, 2001). In contrast, recent studies that distributed patients into groups based on the severity of dyslipidemia found that the incidence of subclinical hypothyroidism was highest in the group with the highest serum cholesterol concentrations (Bindels et al, 1999). Lipid patterns were particularly altered in patients with subclinical hypothyroidism and a serum TSH concentration $>10$ $\mathrm{mU} / \mathrm{L}$, especially in older patients. The discrepancy between these study results may be due 
to the heterogeneity of the populations studied, including differences in TSH concentrations used to define subclinical hypothyroidism and selection criteria based on age, sex, race, smoking history, and insulin resistance. Although hyperinsulinemia may increase the hepatic output of very low-density lipoprotein (VLDL) particles, hypothyroidism may suppress their removal, resulting in a net accumulation of modified lipoproteins. Moreover, LDL particles in subjects with subclinical hypothyroidism have an impaired composition, becoming triglyceride-rich lipoproteins (Duntas \& Brenta, 2012). VLDL remnants and intermediatedensity lipoproteins (IDL) tend to accumulate in the circulation. However, the fractional clearance rates of triglycerides and cholesteryl esters have been reported equal in patients with subclinical hypothyroidism and controls, indicating that lipolysis and the removal of remnant triglyceride rich lipoproteins were normal. Nevertheless, transfer of triglycerides to HDL was lower in subjects with subclinical hypothyroidism than in controls.

In summary, the association between lipid patterns and subclinical hypothyroidism remains unclear. This may reflect differences in population-based studies, as well as differences in age, gender, and ethnicity of the subjects examined. Taken together, these findings have resulted in the formulation of a hypothesis, that there is no TSH cutoff threshold associated with lipids. However, smoking and insulin resistance may play important roles in mediating the effects of subclinical hypothyroidism on serum lipids.

\subsubsection{Mood and cognitive changes}

The relationships between overt thyroid disease and mood impairment and cognitive dysfunction have been described. Overt hypothyroidism is a frequent cause of major depressive disorder, including melancholia, and may lead to reversible dementia. Several relatively small-volume studies found more hypothyroid associated signs and symptoms of mood disorders in individuals with subclinical hyperthyroidism than in euthyroid individuals. Subclinical hypothyroidism may be associated with current depressive symptoms, current major depression and a lifetime history of major depression (Joffe et al, 2012) (Chueire et al, 2007). These studies have reported a higher frequency and/or severity of current depressive symptoms in young or middle-aged adults with subclinical hypothyroidism than in matched euthyroid controls. Moreover, the lifetime prevalence of major depressive disorder has been reported higher in individuals with subclinical hypothyroidism than in euthyroid subjects. These findings suggest an association, but not necessarily a direct causal relationship, between subclinical hypothyroidism and mood disturbance or disorders. Recent studies, however, have suggested that patients with primary major depressive illness have a reduced rate of response to antidepressants and are at greater risk of chronicity of depression if they have comorbid subclinical hypothyroidism.

Although many studies have assessed the relationship between mild hypothyroidism and cognitive dysfunction, these studies have produced somewhat conflicting findings. A recent cross-sectional study of 5865 patients in England aged $\geq 65$ years with no known thyroid disease (168 with subclinical hypothyroidism defined by TSH $>5.5 \mathrm{mIU} / \mathrm{L}$ ) was performed in primary care practices to evaluate the association with mood and cognitive changes. This study found no associations between mild hypothyroidism and cognitive function, depres- 
sion, and anxiety (Roberts et al, 2006). In contrast, evaluation of brain function by functional magnetic resonance imaging (MRI) in patients with overt and subclinical hypothyroidism and euthyroid subjects suggested that working memory, but not other memory functions, was impaired by subclinical hypothyroidism, with impairment more severe in patients with overt hypothyroidism (Zhu et al, 2006). Whereas some studies included only older subjects, other studies include broad ranges of age groups, limiting the conclusions that can be drawn from these data. Perhaps, the most problematic methodological issue in these studies is their reliance on limited measures of cognitive function, especially the Mini Mental State Examination, which provides a very limited assessment of cognition and is likely relatively insensitive to potentially subtle, although clinically meaningful, neuropsychological impairments. This may explain the discrepancy among studies, with some finding and others not finding an association between cognitive alterations and subclinical hypothyroidism (Joffe et al, 2012). Nonetheless, these studies have shown that younger adults with subclinical hypothyroidism may experience mild cognitive abnormalities, generally difficulties with selective attention and new learning. Studies in older adults have found that subclinical hypothyroidism may be associated with deficits in attention, in some aspects of executive functioning, verbal and visual recall, and in reaction time, but these deficits may differ in older and younger individuals, and in men women (Samuels et al, 2008).

The relationship between subclinical hypothyroidism and vulnerability to dementia, especially Alzheimer's disease, has been evaluated in older adults. Although high TSH concentrations were associated with an increased risk of developing Alzheimer's disease or dementia, the association between subclinical hypothyroidism and dementia is unclear. Moreover, the brains of older adults may show differential sensitivity, manifesting as cognitive changes, to small perturbations of the thyroid axis.

In summary, the pattern and severity of mood and cognitive symptoms in patients with subclinical hypothyroidism have not been fully delineated, although both depressive symptoms and depressive syndromes may occur with increased frequency. It is difficult to distinguish euthyroid subjects from patients with subclinical hypothyroidism based on these symptoms. Subclinical hypothyroidism may also be associated with current cognitive impairment and the further risk of cognitive decline. In addition, these symptoms are probably related to disease severity, disease duration, and individual sensitivity to thyroid hormone deficiency. Age may also affect the correlations between subclinical hypothyroidism and mood, cognition, and Alzheimer's disease. Larger randomized controlled studies are necessary to assess the importance of mood and cognitive function in both younger and older age groups, especially in individuals with minimally elevated TSH.

\subsection{Treatment}

There has been much discussion about the screening and treatment of patients with subclinical hypothyroidism. Because of the difficulties in interpreting data from many different sources, the AACE, the US Endocrine Society and the ATA convened a panel in 2002 to formulate evidence-based guidelines for the diagnosis, screening, and treatment of subclinical hypothyroidism (Surks et al, 2004). The consensus panel recommended that patients with 
elevated serum TSH undergo repeat testing, along with a serum fT4 measurement, from 2 weeks to 3 months later. If these tests confirm subclinical hypothyroidism, further evaluation is required, including clinical assessment of signs and symptoms; history taking to determine if the patient had been previously treated for hyperthyroidism (e.g., with radioiodine therapy or partial thyroidectomy), or if there is a family history of thyroid disease; and determination of the occurrence of thyroid enlargement. These patients should also be screened for hyperlipidemia. Although the presence of TPO Ab increases the risk of progression to overt hypothyroidism, the panel found insufficient evidence to recommend for or against obtaining titers because determining the presence of antibodies does not change patient management.

The panel found good evidence that subclinical hypothyroidism is associated with progression to overt hypothyroidism, and fair evidence that serum TSH concentrations $>10 \mathrm{mU} / \mathrm{L}$ are associated with elevated total and LDL cholesterol concentrations. The panel recommended that patients with serum TSH concentrations $>10 \mathrm{mU} / \mathrm{L}$ be treated with levothyroxine sodium (LT4). There is no conclusive evidence that treatment will improve symptoms or associated clinical conditions such as hyperlipidemia; however, because $2-10 \%$ of patients progress to overt hypothyroidism, treatment may prevent symptom development in patients with low fT4 concentrations. Most other guidelines and expert views also recommend treatment of subclinical hypothyroid patients with TSH concentrations $>10 \mathrm{mU} / \mathrm{L}$ (Biondi \& Cooper, 2008) (Gharib et al, 2005) (Khandelwal \& Tandon, 2012) (Vaidya \& Pearce, 2008).

Treatment of asymptomatic patients with serum TSH concentrations between 4.5 and 10 $\mathrm{mU} / \mathrm{L}$ remains unclear. The panel recommended that only pregnant women and women contemplating pregnancy be treated for subclinical hypothyroidism and TSH concentrations $\leq 10 \mathrm{mU} / \mathrm{L}$, based on a possible association between high TSH and subsequent neuropsychological complications in offspring. According to recent ATA guidelines, if a woman is hypothyroid prior to pregnancy, it recommended that her dosage be adjusted so that TSH is below $2.5 \mathrm{mU} / \mathrm{L}$ prior to conception (Stagnaro-Green et al, 2011). This lowers the risk of the TSH elevating in the first trimester. If a woman is diagnosed as hypothyroid during pregnancy, she should be treated without delay, with the goal of restoring her thyroid levels to normal as quickly as possible. In pregnancy, overt hypothyroidism is defined as a TSH above $2.5 \mathrm{mU} / \mathrm{L}$, along with a decreased fT4 level. Even if a woman has normal fT4, if TSH is above $10.0 \mathrm{mU} / \mathrm{L}$ during pregnancy, it is also considered to be overt hypothyroidism. Subclinical hypothyroidism is defined as TSH between 2.5 and $10 \mathrm{mU} / \mathrm{L}$, with a normal fT4 level. During the first trimester, the TSH level should be maintained at a level of between 0.1 and $2.5 \mathrm{mIU} / \mathrm{L}, 0.2$ to $3.0 \mathrm{mIU} / \mathrm{L}$ during the second trimester, and 0.3 to $3.0 \mathrm{mIU} / \mathrm{L}$ in the third trimester. By the time a woman is four to six weeks pregnant, her dose of thyroid medication will usually need to be increased, potentially by as much as 50 percent. A woman with thyroid autoimmunity with positive thyroid antibodies who has normal TSH levels in the early stages of her pregnancy is still at increased risk of becoming hypothyroid at any point in the pregnancy. She should be monitored regularly through the pregnancy for elevated TSH (Stagnaro-Green et al, 2011). 
The panel recommended against routine treatment of patients with subclinical hypothyroidism and serum TSH $\leq 10 \mathrm{mU} / \mathrm{L}$, as available data do not support a clear-cut benefit for early treatment of these patients. However, in a separate consensus statement, these societies recommended that most subclinical hypothyroid patients be considered for treatment, with the key determinant being the clinical judgment of the provider (Gharib et al, 2005). Recently some experts further consolidated the available evidence and recommended LT4 therapy in women who are pregnant, planning pregnancy, have ovulatory dysfunction, or are infertile; as well as in patients with symptoms, goiter, anti-TPO antibodies, and high background cardiovascular risk, including those with hypertension, hypercholesterolemia, insulin resistance or diabetes, isolated diastolic dysfunction, or evidence of impaired endothelial function (Biondi \& Cooper, 2008) (Khandelwal \& Tandon, 2012). Current evidence suggests that middle-aged individuals are more likely to benefit from treatment than elderly individuals. Asymptomatic individuals should undergo repeat thyroid function tests every 6 to 12 months. There is also insufficient evidence to support therapeutic intervention in patients with symptoms of hypothyroidism and serum TSH concentrations between 4.5 and 10 $\mathrm{mU} / \mathrm{L}$. The panel suggested, however, that these patients be started on LT4, with treatment continued only in those experiencing symptomatic benefit (Woeber, 2005).

Thyroid hormone preparations available for treatment of hypothyroidism include levothyroxine sodium (L-thyroxine; LT4) and liothyronine sodium (L-triiodothyronine; LT3). LT4 is synthetically produced but identical to T4 secreted by the thyroid. LT4 is the preferred drug because its administration closely mimics glandular secretion and its conversion to T3 is appropriately regulated by tissues, which maintain a steady and adequate supply. Its long half-life of 7 days allows single daily dose administration and results in only small fluctuations in serum concentrations between daily doses (Khandelwal \& Tandon, 2012) (Woeber, 2005). Serum T4 concentrations peak 2-4 hours after an oral dose and remain above normal for approximately 6 hours in patients receiving daily replacement therapy. LT4 is also recognized to have a narrow toxic to therapeutic ratio, with excess amounts having significant clinical consequences. Adverse effects of over-replacement include the risk of bone loss, especially in postmenopausal women, and increased risk of atrial fibrillation. Transient scalp hair loss can also take place during the first few weeks of LT4 treatment. Allergic reactions have been rarely reported, but these were almost always reactions to dye or other inactive constituents. LT3 is a synthetic form of natural T3 hormone with the same actions as the natural product. It has a half-life of 1 day, thus requiring multiple doses daily. Another disadvantage of LT3 is the increase in serum T3 concentration to supranormal values, up to $250-600 \%$, in the absorption phase, during which many patients report adverse effects, especially palpitations (Wiersinga, 2001) (Woeber, 2005). Treatment with LT3 should therefore be considered only in patients with LT4 maldigestion or malabsorption, as well as in patients who cannot convert T4 to T3 (Celi et al, 2011). Otherwise, it is not intended as sole maintenance therapy in patients with hypothyroidism. Combined treatment with LT4 and LT3 has been attempted to more closely mimic the thyroid secretion patterns of T4 and T3. However, there is no currently available preparation containing both LT4 and LT3 in combination that adequately reproduces the relative quantities of these hormones produced by the human 
thyroid gland. Furthermore, no preparation results in a pattern of sustained release of thyroid hormones similar to that of the human thyroid (Woeber, 2005).

The goal of treatment is to restore the individual to a euthyroid state, with resolution of signs and symptoms of hypothyroidism. Chronic under- or over- replacement is common in clinical practice, with over-treatment occurring in about $20 \%$ of LT4-treated patients (Canaris et al, 2000) (Parle et al, 1993). The rapidity with which the euthyroid state should be attained is dictated by several factors, notably the age of the patient, the duration and severity of hypothyroidism and the presence of other co-morbid conditions, specifically cardiac disease. It has been recommended that LT4 be taken as a single daily dose on an empty stomach at least 30 minutes before breakfast. Individual LT4 requirements are greatly dependent on an individual's lean body mass, rather than on total body weight. Patients with subclinical hypothyroidism and minimal thyroid hormone deficiency may be controlled with daily LT4 dosages as low as $25-50 \mu \mathrm{g}$. After initiation of thyroid hormone therapy, the symptoms and signs of hypothyroidism should be assessed at each follow-up visit. The earliest clinical response to LT4 replacement is usually diuresis and weight loss, leading to mobilization of interstitial fluid as glycosaminoglycans are degraded. Weight loss is predominantly due to fluid loss, and is unlikely to exceed $5 \mathrm{~kg}$, even in obese patients, especially if pre-treatment TSH concentrations were only modestly elevated. Two months after initiating therapy, the minimum time required for the pituitary-thyroid axis to re-set, the dose should be monitored by measuring serum TSH, with or without serum T4. Serum TSH should be maintained in the lower half of the normal range (0.5-2.0 mU/L) (McDermott, 2009).

An important cause of persistently elevated TSH despite an adequate replacement dose of LT4 is patient non-compliance. These patients may have elevated TSH with high normal or elevated fT4, as they may not take LT4 for days and then take several pills a few days before testing. These patients do not require changes in LT4 dose; rather, emphasis should be placed on compliance with therapy and thyroid function tests should be repeated in 3-4 weeks. Other causes of persistently elevated TSH despite an apparently adequate dose of LT4 include malabsorption and interference by drugs. Coeliac disease should be excluded, as it may be present in patients with hypothyroidism because of its autoimmune nature. The presence of heterophilic antibodies in a patient's serum can also result in an artificial elevation of TSH (Khandelwal \& Tandon, 2012).

\section{Conclusion}

Subclinical hypothyroidism is a frequent clinical problem, readily diagnosed by laboratory methods. Since most patients with this condition are asymptomatic, screening is required to detect the condition in most cases. As there is insufficient evidence to support populationbased data, the associations between subclinical hypothyroidism and adverse clinical outcomes remain unclear. Subclinical hypothyroidism was recently reported to be associated with increased risks of cardiovascular disease and heart failure. Subclinical hypothyroidism may or may not be correlated with other systemic conditions, such as changes in lipid me- 
tabolism, mood and cognition, with the clinical significance of these systemic effects after long-term subclinical hypothyroidism being unclear. Findings suggest that these systemic sequelae of subclinical hypothyroidism are associated with increased disease severity, disease duration, age, and individual sensitivity to thyroid hormone deficiency.

The benefits of treatment of subclinical hypothyroidism remain unclear, despite the potential risk of progression to overt disease, and there is no definite consensus on thyroid hormone and TSH cutoff values at which treatment should be contemplated. It has been recommended that all patients with subclinical hypothyroidism with $\mathrm{TSH}>10 \mathrm{mU} / \mathrm{L}$ or showing symptoms be treated, as should all pregnant women with any degree of subclinical hypothyroidism. The benefits of treatment of asymptomatic patients with subclinical hypothyroidism and serum TSH $\leq 10 \mathrm{mU} / \mathrm{L}$ remain unclear. Most expert groups have recommended LT4 therapy for patients with a mild degree of subclinical hypothyroidism (TSH $\leq$ $10 \mathrm{mU} / \mathrm{L}$ ) only if they have symptoms, goiter, anti-TPO antibodies or infertility. Adequacy of treatment is monitored by measuring serum TSH and fT4 concentrations. Special considerations are needed in pregnant women, children, elderly patients and patients with cardiac disease. Under- and over- treatment are common in clinical practice and should be avoided.

\section{Acknowledgment}

All authors including Drs. Lee, and Chung have no conflicts of interest or financial ties to disclose.

\section{Author details}

Jandee Lee ${ }^{1}$ and Woong Youn Chung ${ }^{2}$

1 Department of Surgery, Eulji University College of Medicine, Seoul, South Korea

2 Department of Surgery, Yonsei Univeristy College of Medicine, Seoul, South Korea

\section{References}

[1] Bindels, A., Westendorp, R. G., \& Frölich, M. (1999). The prevalence of subclinical hypothyroidism at different total plasma cholesterol levels in middle aged men and women: a need for case-finding? Clin Endocrinol (Oxf), , 50(2), 217-220.

[2] Biondi, B. (2010). Thyroid and obesity: an intriguing relationship. J Clin Endocrinol Metab , 95(8), 3614-3617.

[3] Biondi, B. (2012). Natural history, diagnosis and management of subclinical thyroid dysfunction. Best Pract Res Clin Endocrinol Metab, , 26(4), 431-446. 
[4] Biondi, B., \& Cooper, . (2008). The clinical significance of subclinical thyroid dysfunction. Endocr Rev, , 29(1), 76-131.

[5] Biondi, B., Palmieri, E. A., \& Klain, M. (2005). Subclinical hyperthyroidism: clinical features and treatment options. Eur J Endocrinol, , 152(1), 1-9.

[6] Boelaert, K., Syed, , \& Manji, N. (2009). Prediction of cure and risk of hypothyroidism in patients receiving 131I for hyperthyroidism. Clin Endocrinol (Oxf), , 70(1), 129-138.

[7] Canaris, G. J., Manowitz, N. R., \& Mayor, G. (2000). The Colorado thyroid disease prevalence study. Arch Intern Med, , 160(4), 526-534.

[8] Cappola, A. R., \& Ladenson, P. W. (2003). Hypothyroidism and atherosclerosis. J Clin Endocrinol Metab, , 88(6), 2438-2444.

[9] Celi, F. S., Zemskova, M., \& Linderman, . (2011). Metabolic effects of liothyronine therapy in hypothyroidism: a randomized, double-blind, crossover trial of liothyronine versus levothyroxine. J Clin Endocrinol Metab, , 96(11), 3466-3474.

[10] Chueire, V. B., Romaldini, J. H., \& Ward, L. S. (2007). Subclinical hypothyroidism increase the risk for depression in the elderly. Arch Gerontol Geriatr, , 44(1), 21-28.

[11] Cooper, D., \& Biondi, B. (2012). Subclinical thyroid disease. Lancet, 3799821. $1142-1154$.

[12] Duntas, L. H., \& Brenta, G. (2012). The effect of thyroid disorders on lipid levels and metabolism. Med Clin North Am, , 96(2), 269-281.

[13] Duntas, L. H., \& Wartofsky, L. (2007). Cardiovascular risk and subclinical hypothyroidism: focus on lipids and new emerging risk factors. What is the evidence? DuntasL. H.WartofskyL. (2007) Cardiovascular risk and subclinical hypothyroidism: focus on lipids and new emerging risk factors. What is the evidence? Thyroid, Vol.17, No.11, pp. 1075-1084, 17(11), 1075-1084.

[14] Gharib, H., Tuttle, R. M., \& Baskin, H. J. (2005). Subclinical thyroid dysfunction: a joint statement on management from the American Association of Clinical Endocrinologists, the American Thyroid Association, and the Endocrine Society. J Clin Endocrinol Metab, , 90(1), 581-587.

[15] Gussekloo, J., van Exel, E., \& de Craen, A. J. (2004). Thyroid status, disability and cognitive function, and survival in old age. JAMA, , 292(21), 2591-2599.

[16] Hollowell, J. G., Staehling, N. W., \& Flanders, W. D. (2001). Serum TSH, T(4), and thyroid antibodies in the United States population (1988 to 1944): National Health and Nutrition Examination Survey (NHANES III). J Clin Endocrinol Metab, , 87(2), 489-499.

[17] Joffe, R. T., Pearce, E. N., \& Hennessey, J. V. (2012). Subclinical hypothyroidism, mood and cognition in older adults: a review. Int J Geriatr. Psychiatry, Epub ahead of print. DOIgps.3796 
[18] Jordan, N., Williams, N., \& Gregory, J. W. (2003). The W546X mutation of the thyrotropin receptor gene: potential major contributor to thyroid dysfunction in a Caucasian population. J Clin Endocrinol Metab, , 88(3), 1002-1005.

[19] Khandelwal, D., \& Tandon, N. (2012). Overt and subclinical hypothyroidism: who to treat and how. Drugs, 72(1), 17-33.

[20] Kratzsch, J., Fiedler, G. M., \& Leichtle, A. (2005). New reference intervals for thyrotropin and thyroid hormones based on National Academy of Clinical Biochemistry criteria and regular ultrasonography of the thyroid. Clin Chem, , 51(8), 1480-1486.

[21] Mc Dermott, M. T. (2009). In the clinic. Hypothyroidism. Ann Intern Med, pp.ITC 61, 151(11)

[22] Meyerovitch, J., Rotman-Pikielny, P., \& Sherf, M. (2007). Serum thyrotropin measurements in the community: five-year follow-up in a large network of primary care physicians. Arch Intern Med, , 167(14), 1533-1538.

[23] Ochs, N., Auer, R., \& Bauer, D. C. (2008). Meta-analysis: subclinical thyroid dysfunction and the risk for coronary heart disease and mortality. Ann Intern Med, , 148(11), 832-845.

[24] Parle, J. V., Franklyn, J. A., \& Cross, K. W. (1993). Thyroxine prescription in the community: serum thyroid stimulating hormone level assays as an indicator of undertreatment or overtreatment. Br J Gen Pract, , 43(368), 107-109.

[25] Razvi, S., Shakoor, A., \& Vanderpump, M. (2008). The influence of age on the relationship between subclinical hypothyroidism and ischemic heart disease: a metaanalysis. J Clin Endocrinol Metab, , 93(8), 2998-3007.

[26] Razvi, S., Weaver, J. U., \& Vanderpump, M. P. (2010). The incidence of ischemic heart disease and mortality in people with subclinical hypothyroidism: reanalysis of the Whickham Survey cohort. J Clin Endocrinol Metab, , 95(4), 1734-1740.

[27] Roberts, L. M., Pattison, H., \& Roalfe, A. (2006). Is subclinical thyroid dysfunction in the elderly associated with depression or cognitive dysfunction? Ann Intern Med, , 145(8), 573-581.

[28] Rodondi, N., den, Elzen. W. P., \& Bauer, D. C. (2010). Subclinical hypothyroidism and the risk of coronary heart disease and mortality. JAMA, , 304(12), 1365-1374.

[29] Samuels, M. H. (2008). Cognitive function in untreated hypothyroidism and hyperthyroidism. Curr Opin Endocrinol Diabetes Obes, , 15(5), 429-433.

[30] Stagnaro-Green, A., Abalovich, M., \& Alexander, E. (2011). Guidelines of the American Thyroid Association for the diagnosis and management of thyroid disease during pregnancy and postpartum. Thyroid, , 21(10), 1081-1125.

[31] Surks, M. I., Ortiz, E., \& Daniels, G. H. (2004). Subclinical thyroid disease: scientific review and guidelines for diagnosis and management. JAMA, , 292(2), 228-238. 
[32] Tunbridge, W. M., Evered, D. C., \& Hall, R. (1977). The spectrum of thyroid disease in a community: the Whickham survey. Clin Endocrinol (Oxf), , 7(6), 481-493.

[33] Vaidya, B., \& Pearce, S. H. (2008). Management of hypothyroidism in adults. BMJ, doi:bmj.a801., 337, a801.

[34] Vejbjerg, P., Knudsen, N., \& Perrild, H. (2006). The association between hypoechogenicity or irregular echo pattern at thyroid ultrasonography and thyroid function in the general population. Eur J Endocrinol, , 155(4), 547-552.

[35] Wiersinga, W. M. (2001). Thyroid hormone replacement therapy. Horm Res, Supp1, , 56, 74-81.

[36] Wilson, G., Curry, R. W., \& Jr , . (2005). Subclinical thyroid disease. Am Fam Physician, , 72(8), 1517-1524.

[37] Woeber, K. A. (2005). Treatment of hypothyroidism. In: Braverman, LE. \& Utiger, RD. (eds). Werner and Ingabar's the thyroid: a fundamental and clinical text. Philadelphia (PA): Lippincott Williams \& Wilkins, , 864-869.

[38] Zhu, D. F., Wang, Z. X., \& Zhang, D. R. (2006). fMRI revealed neural substrate for reversible working memory dysfunction in subclinical hypothyroidism. Brain, , 129(11), 2923-2930. 